\title{
Clinical services used and types of health information sought by low-income Latino patients at a large urban, free clinic
}

\author{
Harmony Raylen Abejuela ${ }^{1,2}$, Shahrzad Bazargan-Hejazi ${ }^{2}$, Kenneth E. Wolf ${ }^{2}$, \\ Eleni Manousogiannakis ${ }^{3}$ and Tony Kuo ${ }^{1,4}$ \\ ${ }^{1}$ University of California, Los Angeles \\ ${ }^{2}$ Charles R. Drew University of Medicine and Science \\ ${ }^{3}$ Venice Family Clinic \\ ${ }^{4}$ Los Angeles County Department of Public Health
}

\begin{abstract}
The present study describes the clinical services used and the types of health information sought by Latino patients at a large urban, free clinic in Los Angeles County, California, USA. A subgroup data analysis of the 2005-2006 Patient Health Assessment Survey, originally administered as part of a clinic quality improvement program, was conducted using a subsample of low-income Latino patients $(\mathrm{n}=301)$ who rely on the targeted clinic for usual care. The descriptive analysis explored health services utilization, including the types of health information sought by members of this clinic population and patient feedback about provider communication strategies that are conducive for mutual decision-making. Overall, healthy eating (61\%) and chronic disease management (58\%) were among the most frequently sought after health information by Latino patients at the clinic. Provider communication strategies, including explaining things in a way that the patient could understand and the use of non-medical language, were suggested by a majority of respondents (> 70\%) as desirable ways to improve the medical encounter experience. Findings from this study may benefit quality improvement efforts in other community health clinics that provide free care to low-income Latino patients with similar backgrounds.
\end{abstract}

(C) 2011 Californian Journal of Health Promotion. All rights reserved.

Keywords: free clinic, patient-centered care, health information seeking, health education

\section{Introduction}

Quality improvement efforts to enhance clinical services often require a broader understanding of patients' health services use and the type of health information they seek, especially among underserved populations or racial/ethnic subgroups who rely on free community health clinics for their usual care (Hardcastle et al., 2008; Schrop et al., 2006). In these populations, disease prevention and lifestyle behavioral modifications (e.g., counseling and brief intervention for diet, physical activity, weight management, smoking, and/or alcohol and drug use) remain some of the most effective and reliable strategies for reducing health risks and medical complications associated with chronic disease (Stead et al., 2008; McAlpine \& Wilson, 2007; Peterson, 2007; Ma et al., 2004; Mellen et al., 2004). Unfortunately, limited access to care, lower educational attainment, poor emphasis on disease prevention, and significant communication inequalities have continued to contribute to worse health outcomes in these populations, as compared to their more affluent cohorts (Kontos et al., 2011; Heaton \& Frede, 2006; Schrop et al., 2006).

Research evidence underscores the importance of communication and having a voice during the medical encounter to facilitate mutual decisionmaking (Tataw \& Bazargan-Hejazi, 2010a). Data from the literature suggest that receiving appropriate health information and directives 
from physicians (Street, 1992) is associated with higher patient satisfaction (Tataw et al., 2010b; Galil et al., 2006) and greater compliance with treatment (Counsell et al., 1993), especially if the information is tailored specifically to reduce linguistic and cultural barriers. Kontos et al. (2011) have argued that racial/ethnic minorities as well as other groups from lower socioeconomic backgrounds often face significant communication barriers that negatively impact their access to and use of accurate health information for improving health. To date, much remains unknown about the patterns of clinical services use and/or the health information seeking behaviors of lower income, minority populations (Hardcastle et al., 2008; Schrop et al., 2006).

To help address this gap, the present study examined the types of clinical services used and the types of health information sought by lowincome Latino patients at a large, urban, free clinic in Los Angeles County, California, USA. The study also explored patient perceptions about communication strategies used by providers at this clinic.

\section{Method}

\section{Study design of the original patient health assessment survey}

The Patient Health Assessment Survey (PAS) was a self-administered survey given to 475 patients who were systematically selected to participate in a quality improvement (QI) program at four primary care services delivery sites in a large urban, free clinic in the western region of Los Angeles County. This multi-site free clinic is among the largest, public-sector free clinics in the United States, with eight service locations spanning Venice, Santa Monica, Mar Vista, and Culver City. The clinic serves more than 24,400 men, women, children, teens, and seniors annually; $97 \%$ of them are low-income and nearly three-quarters are uninsured. More than 106,000 primary care, specialty care, mental health, dental, and health education visits occur each year. The PAS was conducted during the period December 2005 to January 2006. Although most of the activities of the QI program are continuous, the patient survey component is only repeated periodically. Patients were invited to participate in the 20052006 PAS if they met the following inclusion criteria: 1) aged 20 years and older and 2) had at least one prior visit to the clinic in the past 12 months. Participation in the survey was voluntary. Prospective respondents were approached in the waiting rooms of the clinic sites, typically during adult medicine clinic visits. Among patients who were approached and invited to participate, more than half $(53 \%)$ consented to the survey and completed the questionnaire.

The self-administered PAS questionnaire contained 94-multiple-item questions that examined patient socio-demographics, personal health risk behaviors, the types of health information patients seek, the use of clinical services, and patient perceptions about the quality of care at the clinic. The questionnaire also evaluated patient satisfaction ratings of the care they received at the clinic during the preceding 12 months. The question items were adapted from several sources, including prior clinic patient health assessment surveys and other national as well as local population-based surveys such as the National Health Interview Survey (NHIS), the Behavioral Risk Factor Survey (BRFS), and the Los Angeles County Health Survey (LACHS). Details about these surveys, including their design, methods, instrument construction process, and question items, are described elsewhere (NHIS, http://www.cdc.gov/nchs/nhis.htm; BRFS, http://www.cdc.gov/brfss/; LACHS, http://publichealth.lacounty.gov/ha/hasurveyintr o.htm). The design and implementation of the 2005-2006 PAS were informed and guided by the literature on health education and framework on patient health empowerment (Hubley, 2006). The questionnaire was available in both English and Spanish.

\section{The present study}

The present study was a cross-sectional analysis of the original PAS dataset $(n=475)$. A subgroup analysis was conducted, focusing on data for 301 Latino patients who participated in 


\section{Table 1}

\section{Percentage of survey respondents who were seeking and/or using the types of health} information and clinical services listed $(n=301)$

\begin{tabular}{|c|c|c|c|c|c|}
\hline $\begin{array}{l}\text { Types of Health Information } \\
\text { Sought }\end{array}$ & $\%$ & $\begin{array}{l}\text { Types of Health Services } \\
\text { Used }\end{array}$ & $\%$ & $\begin{array}{l}\text { Types of Counseling Services } \\
\text { Received }\end{array}$ & $\%$ \\
\hline Choose healthy foods & 61 & General medical services & 59 & Fruits \& vegetables & 81 \\
\hline Keep a healthy weight & 60 & $\begin{array}{l}\text { Reproductive care (e.g., family } \\
\text { planning) }\end{array}$ & 19 & Exercise & 79 \\
\hline Healthy cooking & 59 & Prenatal classes & 12 & Quit smoking & 30 \\
\hline Chronic disease management $^{\dagger}$ & 58 & Nutrition health education & 11 & Alcohol use & 16 \\
\hline Deal with stress & 48 & $\begin{array}{l}\text { Women, Infants, and Children } \\
\text { (WIC) }\end{array}$ & 10 & Illicit drug use & 9 \\
\hline Be a better parent & 40 & Diabetes classes & 5 & & \\
\hline Deal with depression & 37 & Weight loss/management education & 6 & & \\
\hline Interest in relaxation therapy & 31 & Psychiatry services & 5 & & \\
\hline Interest in herbal medicine & 29 & Social work counseling & 5 & & \\
\hline Interest in yoga & 26 & Healthy newborn services & 3 & & \\
\hline Interest in chiropractic & 23 & Parenting classes & 3 & & \\
\hline Interest in acupuncture & 21 & Weight Busters & 3 & & \\
\hline $\begin{array}{l}\text { Deal with alcohol or drug } \\
\text { problems }\end{array}$ & 17 & "Mommy and Me" classes & 1 & & \\
\hline Deal with domestic violence & 17 & & & & \\
\hline
\end{tabular}

the original survey. Data analyses examined patients' self-reported demographic profiles, health risk behaviors, interests in the different types of health information available, and the use of clinical services at the clinic, including the Women, Infants, and Children (WIC) program, prenatal classes, diabetes self-care programs, and lifestyle-related counseling on diet, physical activity, cigarette/tobacco use, and/or alcohol and other drug use.

\section{Key health services indicators}

Key health services indicators examined in the analysis included the 14-item (Yes $=1$, No $=0$ ) question on health information seeking (HIS) or the types of health information respondents reported having interests in (Table 1); the 13item (Yes $=1$, No $=0$ ) question on clinical services used (CSU) (Table 1); the 5-question battery assessing provider counseling on health habits (Table 1); and 13-item (Yes =1, No = 0) composite question querying patients about their perceptions of and experiences with provider communication strategies, and what they think providers can do differently to improve patientprovider communication (PCS) (Table 2).

Other health-related variables examined in the analysis included: patients' perceived physical (1-item) and mental (1-item) health status, each measured as excellent $=5$, very good $=4$, good $=3$, fair $=2$, and poor $=1$; cigarette/tobacco use in the past 3 months, which was measured as daily or almost daily $=4$, weekly $=3$, monthly $=$ 2 , once or twice $=1$, or not smoked cigarettes in the past 3 months $=0$; and problem drinking, asking male respondents "how many times in the past 30 days have you had 5 or more drinks of beer, wine or liquor in a day?", or for women "4 or more drinks of beer, wine or liquor in a day?". Respondents were also asked if they have had or currently have any chronic medical conditions, such as diabetes, asthma, arthritis, hypertension, and/or depression (Yes $=1$, No $=$ $0)$. Demographic variables included age, gender, education, employment, country born, number of years lived in the United States, and language spoken at home. 
Table 2

\section{Percentage of survey respondents who reported or suggested that the listed types of provider communication strategy could help or have helped them better understand their health issues.}

\begin{tabular}{lr}
\hline Provider Communication Strategy & $\%$ \\
\hline $\begin{array}{l}\text { Provider explained things in a way the patient could } \\
\quad \text { understand }\end{array}$ & 76 \\
Provider repeated instructions during the visit & 51 \\
Provider used non-medical words and language & 31 \\
Provider asked patient about what was said & 25 \\
Provider used simpler words to make sure the patient & 17 \\
$\quad$ understood & 12 \\
Provider asked patient to repeat instructions & 12 \\
Provider talked slowly to make sure the patient & \\
$\quad$ understood the instructions & 11 \\
Provider gave information and materials that were & \\
$\quad$ easy to read & 10 \\
Provider spent time answering questions & 8 \\
Provider demonstrated patience & 7 \\
Provider took more time with the patient & \\
Provider used/did other things to communicate & 6 \\
$\quad$ information during the visit & \\
Provider did anything to make sure patient understood & 2 \\
Provider drew pictures during the visit & 1 \\
\hline
\end{tabular}

\section{Data analysis}

Descriptive and bivariate analyses were performed on key health services indicators used in the present study. Patient-reported frequencies of services use and suggestions for ways to improve clinical services were evaluated to generate frequencies for of each of the indicators (e.g., HIS, CSU, PCS, receipt of counseling).

Pearson correlation coefficients were calculated to explore the relationships between the indicators (e.g., PCS and CSU). The sum of the scores for the related items from each of these indicators was calculated. Higher values were interpreted as higher patient reports of services used and/or desired outcomes (i.e., patient preference of what they would like providers to do differently when communicating to them about care management plans).

A p-value of $<0.05$ was considered to be statistically significant in the study analyses. All analyses were conducted using the SPSS Version 16.0 statistical software package (SPSS, Inc.; Chicago, IL). The study received exemption approval from the Institutional Review Boards at the Charles R. Drew University of Medicine and Science and the UCLA David Geffen School of Medicine.

\section{Results}

The majority of the study respondents (Latino patients) were female (79\%), unmarried, and less than 30 years of age. The mean age was $44.6+/-14.3$ years old (range: 20 to 89 years). Sixty-eight percent attained at least a high school education; $57 \%$ were employed, $92 \%$ were born in Mexico; and 68\% had lived in the United States for more than 11 years. A majority spoke Spanish only (51\%), while $15 \%$ spoke both English and Spanish. Only 21\% reported having health insurance.

Approximately $41 \%$ rated their overall physical health as poor/fair, compared to only $15 \%$ who rated their overall mental health as poor/fair. Twenty percent of the respondents reported excessive (daily or almost daily) cigarette/tobacco use; $21 \%$ reported problem use with alcohol ( $>5$ drinks per day for men and $>4$ drinks per day for women in the past 30 days). Almost half (47\%) indicated that their providers have told them they have at least one of the following chronic conditions: hypertension, heart disease, or diabetes.

Of the 14 health-related topics listed in HIS, healthy eating, maintenance of a healthy weight, and managing chronic diseases were among the top five most frequently sought after health information; collectively, at least $58 \%$ of the survey respondents reported having interest in these topics. Many respondents also expressed interest in stress management, depression care, and alternative and complementary medicine (e.g., chiropractic, acupuncture, yoga, herbal medicine).

Survey respondents frequently reported using general medical services; reproductive care (e.g., family planning, infant care, birth control/oral contraceptive use, etc); and a range of health 
education, counseling and other preventive services. Table 1 shows patient-reported use of clinical services at the clinic for the period 20052006.

Respondents provided feedback on what they thought providers have done well or could do differently to help facilitate better patientprovider communication and mutual decisionmaking. They reported or suggested an array of proactive communication strategies including explaining things in ways that could be understood and using non-medical language (Table 2). In correlation analysis, these communication strategies were positively correlated with the use of health promotion services by these patients at the clinic $(\mathrm{r}=0.41$, $\mathrm{p}<.001)$. This relationship between PCS and CSU, however, is likely confounded by other contributing factors such as the type and nature of the health promotion services being offered, patients' co-morbidities, organizational structure/characteristics, and patient visit patterns.

\section{Discussion}

In the present study, we examined self-reported clinical services used and the types of health information sought by Latino patients at a large, urban, free clinic in Los Angeles County. The study yielded two interesting findings that may have clinical practice implications for this underserved population.

First, a substantial percentage of Latino patients who participated in the 2005-2006 PAS reported desiring more information about health promotion and self-care strategies for managing chronic disease. They were interested in such alternative treatment modalities as acupuncture, chiropractic, yoga, and stress management (Table 1). Together, these results suggest an unmet demand for self-care information in this population. Kontos and co-workers (2011) have previously studied similar instances of unmet demands or needs in minority populations, concluding that access barriers to quality health information can exacerbate disparities in health outcomes among disadvantaged populations. Communication inequalities such as these and others can perpetuate gaps in health knowledge and beliefs among lower income individuals (i.e., less empowered to seek out accurate information and to act), as compared to those from a more affluent background (Kontos et al., 2011). According to Johnson and colleagues (2004), the patient's voice can be strengthened through training to help address these communication inequalities.

Second, a substantial percentage of Latino patients who participated in the survey reported a preference for more proactive provider communication strategies (e.g., use of nonmedical language, simple explanations, more time spent explaining care management plans, etc.). As communication strategies are generally not costly nor labor-intensive to implement, institutional investments in ongoing provider training and in building a practice environment which supports the usage of these types of strategies for empowering patients and for facilitating mutual decision-making (e.g., allowing more time for providers to talk to their patients and for patients to ask questions) would seem reasonable and feasible to do (Greene \& Yedidia, 2005; Chakravarthy et al., 2002). Evidence from organizational research suggests that if system-level changes are undertaken with the support of managers and staff, the adoption of these types of patient-centered communication strategies into routine clinical practice could face minimal institutional resistance and result in salutary impacts on patient health outcomes in the long-term (Greene \& Yedidia, 2005; Chakravarthy et al., 2002; Committee on Quality of Health Care in America, 2001). Use of patient-centered communication is timely for patient populations such as the population described in the present study, as the reported rates of poor physical and mental health and risky behaviors, including smoking and problem drinking, are relatively high in this subgroup.

\section{Study Limitations}

The generalizability of the study findings is subject to at least three limitations. First, the study sample is not representative of the general population in Los Angeles County or of the insured Latino groups. Instead, the sample is 
more representative of uninsured or underinsured Latino patients who rely upon public sector clinics in Los Angeles County for selected clinical services. Second, self-report and selection bias may have overestimated or underestimated the respondents' interests and their reported use of clinical services. Third, the study design may be inadequate for evaluating other factors which may influence health information seeking behaviors, patient preferences and satisfaction, and quality of clinical services delivered. For example, the original survey did not specifically ask about health literacy or cultural tailoring of health education materials for Latino patients. These two factors, along with others, are likely to be just as important as health information availability or use of proactive provider communication strategies for enhancing quality of care in this low-income clinic population.

\section{Acknowledgments}

The study was partly supported by the Title III Part B "Strengthening Historically Black Graduate Institutions" Grant P031B070062, and by the NIH-NCRR-U54 RR026138-03.

\section{References}

Chakravarthy, M.V., Joyner, M.J. \& Booth, F.W. (2002) An obligation for primary care physicians to prescribe physical activity to sedentary patients to reduce the risk of chronic health conditions. Mayo Clinic Proceedings, 77(2), 165-173.

Committee on Quality of Health Care in America, Institute of Medicine (IOM). Crossing the quality chasm: a new health system for the 21st century. Washington D.C.: National Academy Press, 2001.

Counsell, A.M., Geddis, D.C. \& Smith, A.R. (1993) Parental perceptions of information about medication prescribed for their children. The New Zealand Medical Journal, 106(956), 205-206.

Galil, A., Bachner, Y.G., Merrick, J., Flusser, H., Lubetzky, H., Heiman, N. \& Carmel, S. (2006) Physician-parent communication as predictor of parent satisfaction with child development services. Research in Developmental Disabilities, 27(3), 233-242.

Greene, J. \& Yedidia, M.J. (2005) Provider behaviors contributing to patient self-management of chronic illness among underserved populations. Journal of Health Care for the Poor and Underserved, 16(4), 808-824.

Hardcastle S., Taylor, A., Bailey, M. \& Castle, R. (2008) A randomised controlled trial on the effectiveness of a primary health care based counselling intervention on physical activity, diet and CHD risk factors. Patient Education and Counseling, 70(1), 31-39.

Heaton, P.C. \& Frede, S.M. (2006) Patients' need for more counseling on diet, exercise, and smoking cessation: results from the National Ambulatory Medical Care Survey. Journal of the American Pharmacists Association, 46(3), 364-369.

Hubley, J. (2006) Patient education in the developing world - a discipline comes of age. Patient Education and Counseling, 61(1), 161-164.

Johnson, R.L., Saha, S., Arbelaez, J.J., Beach, M.C. \& Cooper, L.A. (2004). Racial and ethnic differences in patient perceptions of bias and cultural competence in health care. Journal of General Internal Medicine, 19(2), 101-110.

Kontos, E.Z., Emmons, K.M., Puleo, E. \& Viswanath, K. (2011) Determinants and beliefs of health information mavens among a lower-socioeconomic position and minority population. Social Science \& Medicine, 73(1), 22-32.

Ma, J., Urizar, G.G., Jr., Alehegn, T. \& Stafford, R.S. (2004) Diet and physical activity counseling during ambulatory care visits in the United States. Preventive Medicine, 39(4), 815-822.

McAlpine, D.D. \& Wilson, A.R. (2007) Trends in obesity-related counseling in primary care: 1995-2004. Medical Care, 45(4), 322-339.

Mellen, P.B., Palla, S.L., Goff, D.C. \& Bonds, D.E. (2004) Prevalence of nutrition and exercise counseling for patients with hypertension. United States, 1999 to 2000. Journal of General Internal Medicine, 19(9), 917-924. 
Peterson, J.A. (2007) Get moving! Physical activity counseling in primary care. Journal of the American Academy of Nurse Practitioners, 19(7), 349-357.

Schrop, S.L., Pendleton, B.F., McCord, G., Gil, K.M., Stockton, L., McNatt, J. \& Gilchrist, V.J. (2006) The medically underserved: who is likely to exercise and why? Journal of Health Care for the Poor and Underserved, 17(2), 276-289.

Stead, L.F., Bergson, G. \& Lancaster, T. (2008) Physician advice for smoking cessation. Cochrane Database System Rev. 2, CD000165.

Street, R.L. (1992) Communicative styles and adaptations in physician-parent consultations. Social Science \& Medicine, 34(10), 1155-1163.

Tataw, D.B. \& Bazargan-Hejazi, S. (2010a) Impact of the health services utilization and improvement model (HUIM) on self efficacy and satisfaction among a Head Start population. Journal of Health and Human Services Administration, 33(2), 228-256.

Tataw, D.B., Bazargan-Hejazi, S. \& Patel, P. (2010b) Voice and trust as predictors of parental satisfaction with child health care among a Head Start sample. American Journal of Health Studies, 25.3.

Author Information

Harmony Raylen Abejuela, MD

University of California, Los Angeles

Charles R. Drew University of Medicine and Science

Shahrzad Bazargan-Hejazi, PhD

Charles R. Drew University of Medicine and Science

Kenneth E. Wolf, PhD

Charles R. Drew University of Medicine and Science

Eleni Manousogiannakis, MPH

Venice Family Clinic

Tony Kuo, MD, MSHS*

University of California, Los Angeles

Los Angeles County Department of Public Health

Email: tkuo@mednet.ucla.edu

* corresponding author 